incidence, trends and type of occupational disease (OD) and ii) incapacity for work due to ODs. It is hypothesized that workers in lower and higher SEP still differ in health disparities from an occupational perspective.

Methods From a Dutch cohort of occupational physicians (OPs), ODs assessed by OPs were retrieved over a seven year period (2010-2016) for lower and higher SEP groups. Incidence and type of $\mathrm{OD}$ and incapacity for work were determined for the total number of ODs and six frequently occurring ODs. Trends in incidence were estimated using a multilevel negative binominal regression model.

Result In total, 54 per 100,000 workers in elementary occupations, machine operating and assembly jobs, as well as managerial and intellectual jobs during 2016 had an OD diagnosed and reported by an OP, from which 98 per 100,000 were for lower SEP, and 36 per 100,000 for higher SEP. Among the lower SEP, musculoskeletal disorders (37\%) and noise-induced hearing loss (NIHL) (32\%) comprised two-thirds of the OD diagnoses. Among the higher SEP, distress/burnout comprised $60 \%$ of the OD diagnoses, with an increasing trend $(6 \%$; $95 \%$ CI: 3\%$8 \%)$. Incapacity for work due to work-related low back disorders (69\% vs 9\%) and shoulder-, arm- and wrist disorders (89\% vs 47\%) differed significantly between workers in lower compared to higher SEP.

Discussion Occupational diseases occur at a 2.7 higher incidence rate for workers in lower SEP compared with higher SEP. Incapacity for work due to work-related musculoskeletal disorders are higher for workers in lower SEP compared with higher SEP, suggesting fewer opportunities to modify work tasks and working circumstances for lower SEP. Psychosocial risk factors constitute the greatest problem for workers in higher SEP, resulting in distress/burnout, accompanied by incapacity for work.

\section{E.4 BRIDGING THE GAP BETWEEN CLINICAL CASES AND EPIDEMIOLOGICAL EVIDENCE}

${ }^{1}$ Lode Godderis*, 'Jelena Bakusic, 'Sara Pauwels, ${ }^{2}$ Henk Van der Molen, ${ }^{2}$ Annet Lenderink. ${ }^{1} \mathrm{KU}$ Leuven, Leuven, Belgium; ${ }^{2}$ AMC/University of Amsterdam, Amsterdam, The Netherlands

\subsection{6/OEM-2019-EPI.135}

One of the current gaps in the prevention of work-related diseases (WRDs) is the missing link between the assessment of occupational hazards introduced at the workplace, clinical alerts, epidemiological studies and policy actions. Alert systems aim to bridge this gap, by collecting information on diseases and exposures to raise alerts to different stakeholders and trigger timely prevention.

This project started with a review of international alert systems to identifying good practices and learning about prerequisites, drivers and obstacles to implement alert and sentinel systems. Next, the findings led to the implementation of an integrated approach consisting of alert systems on three levels in the Belgian context:

1. Signal detection and assessment: suspected clinical cases of new WRDs (new exposure-disease combinations) can be reported though an online platform called 'SIGNAAL'. Each reported case is followed by an extensive assessment of exposure- and work-relatedness performed by clinical experts. Since the start, 22 cases have been reported to the platform.

2. Signal strengthening though a network of occupational health physicians. This sentinel approach has been introduced though the PROBE system, in which 47 occupational physicians participated. During the periodic health examinations of workers, the physicians filled in a web survey regarding occupational exposure of a random sample of workers to 22 selected hazardous chemicals during the last working week. Results of the first study showed that $47 \%$ of workers were exposed to at least one chemical product from the list, with diesel exhaust being the most frequently reported substance $(n=91 ; 14 \%$ of workers).

3. Alerts to public health authorities are mainly communicated though collaboration with Federal Public Services and Federal Agency for Occupational Risks.

These agencies provide support in the maintenance of systems and in turn, data from the systems is used as input for potential preventive strategies on company and societal level.

\section{Occupational Health in Women}

\section{O6A.1 AGGREGATION OF WORK-RELATED HEALTH PROBLEMS THROUGHOUT WORKING-LIFE IN A POPULATION-BASED SAMPLE OF WOMEN}

1Joana Amaro*, 1,2Teresa Monjardino, 1,2Raquel Lucas, 3,4,5Mònica Ubalde-Lopez, 1,2Henrique Barros. 'EPIUnit - Instituto de Saúde Pública, Universidade do Porto, Rua das Taipas, $n^{\circ}$ 135, 4050-600 Porto, Porto, Portugal; ${ }^{2}$ Departamento de Ciências da Saúde Pública e Forenses e Educação Médica, Faculdade de Medicina, Universidade do Porto, Porto, Portugal; ${ }^{3}$ CISAL - Center for Research in Occupational Health, Pompeu Fabra University (UPF), Barcelona, Spain; ${ }^{4}$ CIBERESP, CIBER in Epidemiology and Public Health, Madrid, Spain; ${ }^{5}$ MIM (Hospital del Mar Medical Research Institute), Barcelona, Spain

\subsection{6/OEM-2019-EPI.136}

Introduction Multimorbidity potentiates a spectrum of adverse health outcomes that surpass those of individual diseases. However, little is known on disease aggregation related to occupational exposures.

Objective To assess the impact of life course occupational experience on health problem aggregation.

Methods We evaluated 4330 adult women at the 10-yearold follow-up wave of the population-based birth cohort Generation XXI, using a self-administered version of the Labour Force Survey item inquiring whether they had ever had a 'physical or mental health problem that was caused or made worse by your current work or any previous work' with 11 close-ended and one open-ended response options. Disease aggregation was assessed using principal components (PC) analysis and component scores were summarized by sociodemographic, anthropometric and workrelated characteristics.

Results We identified five components to describe disease aggregation which accounted for $54.7 \%$ of observed variance. PC1 gathered all items on musculoskeletal disorders (back, upper and lower limb); participants with lower educational level, higher BMI, blue-collar jobs, working in the private sector, and with a history of occupational accidents scored higher in this component. PC2 gathered the item on mental disorders 
(including anxiety and depression) together with headache and/or eyestrain, and showed higher scores among women with higher educational level, white-collar jobs, and in the public sector. PC3 included the item on other disorders (comprising neurological, endocrine, autoimmune and voice-related) with some loading from digestive and hearing disorders, and showed higher scores in older and blue-collar workers. PC4 grouped respiratory with infectious diseases; it associated with working in the public sector and reporting work-related accident history. PC5 was composed by circulatory system conditions and associated with former smoking, higher BMI, and white-collar jobs.

Conclusion Work-related diseases aggregated in five distinct components, supporting the need for a shift from a disease-by-disease approach towards a worker-centered approach.

\section{O6A.2 BREAST CANCER RISK IN 40.000 DANISH WOMEN BY INDUSTRY}

Julie Elbæk Pedersen*, Johnni Hansen. The Danish Cancer Society Research Center, Copenhagen, Denmark

\subsection{6/OEM-2019-EPI.137}

Background Occupational exposures have been hypothesized as contributors to breast cancer. Nevertheless, studies exploring the risk of breast cancer by occupation or industry generally show somewhat inconsistent results. In addition to small study size and lack of lifetime work history, potential confounders, such as reproductive history, have often not been taking into account and mortality has often been used as outcome. We conducted a nationwide register based nested case-control study in order to explore associations between occupation and incidence of breast cancer, including reproductive history.

Methods A total of 38,347 employed women born in Denmark during the period 1946 to 1995 , and diagnosed with breast cancer 1970-2015 were identified in the nationwide Danish Cancer Registry. Five control subjects, work active and born in Denmark, and free of breast cancer at the date of diagnosis of the corresponding case, were chosen randomly from the Danish Civil Register. Using the unique personal identification number assigned to all residents in Denmark, we obtain lifetime employment history from the nationwide Pension Fund Register, which on an individual level has kept detailed information on all employments in Denmark since 1964. Lastly, information on reproductive factors, and socioeconomic status was retrieved from the Danish Civil Register. Odds ratios (ORs) with 95\% confidence intervals (CIs) were estimated by conditional logistic regression analysis.

Preliminary results: Risk elevations were e.g. seen in the military $(2.1 ; 1.49-2.90)$, printing $(1.6 ; 1.22-2.06)$, airline transportation $(2.4 ; 1.33-4.47)$, automobile service $(1.3 ; 95 \%$ CI 1.12-1.49), laundries (2.4; 1.48-3.79), hairdressing (1.9; $1.11-3.23)$ and paint factories (1.6; 95\% CI 1.22-2.06). Risk reductions were seen in farming $(0.4 ; 0.21-0.59)$, window cleaning $(0.6 ; 0.44-0.84)$ and building and carpentry $(0.8$; 0.70-0.98).

Conclusions The study suggests that breast cancer risk varies significantly by industry and that occupational exposures, including night-shift work, solvents and outdoor work, may play a possible role.

\section{A.3 IMPLICATIONS OF THE WORKPLACE GENDER COMPOSITION FOR DEPRESSION-RELATED SERVICE UTILIZATION: A RETROSPECTIVE COHORT STUDY OF U.S. ALUMINUM WORKERS}

${ }^{1}$ Holly Elser*, ${ }^{2}$ David Rehkopf, ${ }^{1}$ Patrick Bradshaw, ${ }^{3}$ Carmen Brick, ${ }^{3}$ Daniel Schneider, ${ }^{4}$ Ellen Eisen, ${ }^{5}$ Mark Cullen. ${ }^{1}$ UC Berkeley School of Public Health, Division of Epidemiology, Berkeley, USA; ${ }^{2}$ School of Medicine, Department of Medicine, Division of Primary Care and Population Health, Stanford University, Stanford, USA; ${ }^{3}$ UC Berkeley, Department of Sociology, Berkeley, USA; ${ }^{4}$ UC Berkeley School of Public Health, Division of Environmental Health Sciences, Berkeley, USA; ${ }^{5}$ Center for Population Health Sciences, Stanford University, Palo Alto, 94305

\subsection{6/OEM-2019-EPI.138}

Introduction Past research finds that both male and female workers in male-dominated workplaces experience decreased job satisfaction and increased work-related stress. Female workers in particular are more likely to experience gender discrimination, sexual harassment, and social isolation. Nevertheless, systematic evidence regarding the association between workplace gender composition and worker mental healthcare utilization remains limited.

Methods Using data from the American Manufacturing Cohort Study (AMC), we examined the association between workplace gender composition and risk of depression-related outpatient visits among hourly workers employed at one of 32 U.S. aluminum plants between 2003 - 2013.Using generalized additive models (GAM) with a logit link, we modeled risk of depression-related outpatient visits as a function of annual workplace gender composition (i.e. percent women) and covariates (baseline age, race, sex, plant, and calendar year). We captured potential nonlinear relationships using cubic smoothing splines. We used g-computation to summarize the counterfactual risk of depression-related outpatient visits under three hypothetical interventions wherein women comprised at least 10,20 , or 30 percent of the workforce at all plants for all years of followup. We compared these counterfactual risks to the risk under no intervention (the observed data).

Results Across all 32 U.S. plants, the final study sample included 5,279 female and 24,124 male hourly workers. We observed significant reductions in the number of depressionrelated outpatient visits when women comprised at least $20 \%$ $(\mathrm{RD}=-6.2,95 \% \mathrm{CI}-8.75,-3.65)$ and $30 \%(-14.6 ; 95 \% \mathrm{CI}-$ $19.0,-10.2)$ of the workforce. Findings were consistent within subgroups of male and female workers.

Conclusion The gender composition of industries, occupations, and workplaces is an important expression of social structure. We find that modest increases in the percent women employed in otherwise male-dominated work environments can lead to measurable reductions in the frequency of depression-related outpatient visits among male and female hourly workers.

\section{A.4 RISK OF POSTMENOPAUSAL BREAST CANCER AND OCCUPATIONAL EXPOSURE TO CHEMICALS IN SWEDISH WOMEN}

\footnotetext{
${ }^{1,2}$ Per Gustavsson*, 'Cecilia Videnros, 1,2Pernilla Wiebert, 'Nils Plato, ${ }^{3}$ Signe Borgquist, ${ }^{3,4}$ Jonas Manjer, 1,2Maria Albin, ${ }^{1} J$ Jenny Selander. 'Institute of Environmental Medicine, Karolinska Institutet, Stockholm, Sweden; ${ }^{2}$ Centre for Occupational and Environmental Medicine, Stockholm County Council, Stockholm, Sweden; ${ }^{3}$ Department of Clinical Sciences, Malmö, Lund University, Malmö, Sweden; ${ }^{4}$ Department of Surgery, Skåne University Hospital, Malmö, Sweden
}

10.1136/OEM-2019-EPI.139 